\title{
A Proposed Mobile based Payment System (Quickpay) for Nigerian Universities
}

\author{
Agwu Chukwuemeka Odi ${ }^{1 *}$, Odegwo Ifeanyi ${ }^{2}$, Achi Ifeanyi Isaiah ${ }^{3}$, Igwe Joseph Sunday' and Alo \\ Uzoma Rita ${ }^{4}$ \\ 'Department of Computer Science, Ebonyi State University. Abakaliki, Nigeria; \\ emeka2010@yahoo.com, igwejoesun@yahoo.com \\ 2Department of Computer Science, Renassiance University, Enugu, Nigeria; writedegwo53@yahoo.com \\ ${ }^{3}$ Department of Maths/Comp Sci/Stats/Info, Alex Ekwueme University, Ndufu-Alike, \\ Nigeria; bishopachai@yahoo.com \\ ${ }^{4}$ Alex Ekwueme University, Ndufu-Alike, Nigeria; auzomarita@yahoo.com
}

\begin{abstract}
Objectives: In this research work, we proposed and designed a QR code, 2FA authentication based mobile payment system (Quickpay) for Nigerian universities with focus on Ebonyi State University, Abakaliki. Quickpay mobile payment system will aide student in the payment of all the necessary fees required by the university. Methods and Analysis: We deployed object oriented analysis and methodology where we utilized Unified Modelling Language (UML) in analyzing and modelling the new system. Then, the methodology facilitated the use of competent high level programming tool (PHP programming language) for the development of the software (Quickpay mobile app) after detailed system analysis and design. Findings: When using the system proposed in this study, the different security model used in design of mobile payment solution which was seamlessly integrated into the system that will ensure authorized access. This model deploys 2FA authentication system at the initial stage of verification and an SMS token (request for SMS code and User ID) at the second stage of verification to guarantee user access to the system. On successful authentication, the user uses phone to scan the QR code and send an SMS using USSD code and payment code user will click on generate invoice in the form QR code, the user will use a mobile generated form of the invoice to complete the payment transaction. Application Improvements: Despite the QR code system as deployed in most mobile payment system, Quickpay is designed to seamlessly interface with the university portal while utilizing several layers of possession factor authentication methods. This includes an SMS module to generate a security token for authentication and the $2 \mathrm{FA}$ authentication system.
\end{abstract}

Keywords: Mobile Payment, Mobile Technology, QR code, 2FA Authentication, USSD Code

\section{Introduction}

Due to the rapid increase in the use of mobile technology such as mobile phone, PDAs and other mobile device etc, to communicate, send and receive message, perform several vital activities: business companies, industries and institutions have discovered a new way of adopting mobile device to transact business in terms of payment for goods and services. Mobile devices have become part of people's everyday life. It has the potential to transform the ways of conducting common commercial activities. Mobile phones have displaced many familiar devices such as cameras and satellite Nav (satellite Navigation) systems. The camera and satellite Navigation are now integrated into mobile phone as you don't need to carry many devices on yourself.

Because of this exceptional features and mobile phone being available in the hand of many individual, mobile phones has been considered as a commercial viable instrument for carrying out various types of activities,

*Author for correspondence 
especially financial transactions ${ }^{1}$. It is expected that the growth of mobile payment will increase rapidly in the next few decades 2 .

As more and more consumers are equipped with the use of mobile phone to make payment, retailers may need to start considering adding mobile payment to the available payment options. However, since m-payment provides a number of benefits such as speed, convenience and payment for goods and services independent of time and location, it is expected to generate millions of revenues ${ }^{3}$.

Mobile payment is a payment method where a mobile device is needed for initiating and confirming a payment. This could be further explained to be the transfer of an electronic means of payment from the payer to the payee through the use of an electronic payment instrument, which is a mobile device, held by at least one participant, who is not bound to any place and send, receive information over a wireless link ${ }^{4}$.

Mobile payment is fast growing and business transactions are done with ease based on the agreement between the customer and vendor. It has given a new phase of life to the general public, the mobile phones are not only used for oral communication rather can serve several purposes. The mobile payments produce big impacts on both the economies, developed and developing nations ${ }^{5}$.

It improves the standard at which people live by displacing the cash economy and increasing the electronic transactions. Mobile based payment system has changed the cycle of economy in the developed world and in the developing nation where transaction done with mobile phone have overtaken the traditional payment system, user make payment at their convenience ${ }^{6}$. The Banking sector today uses a mobile device to send alert, to update the customer on current financial status as regards to account balance. Banks Use a Short Code (USSD) to enable users transfer money to a vendor, check balance, recharge airtime, pay bills and any other added services rendered to their customers.

Quickpay mobile payment system is designed to utilize mobile phone for ease of payment of services to an individual, business organization, schools, churches and the government. However it is designed for Nigerian Universities to enable students make payment at their own pace. Quickpay will be used by student to pay their school fees, departmental dues, pg forms, acceptance fees, faculty fees, medical fees and transcript etc. Quickpay will be uploaded to Google play store as soon as is tested and certified so as to enable the entire student download and through the options provided make their payment as they deemed. Quickpay will replace the traditional method of going to the bank to pay, obtaining a teller after payment and then going to the bursary unit to change the teller into receipt after one to two weeks pending when the financial institution will supply the details concerning individual payment. Quickpay mobile payment system will provide the university with report on daily basis without stress. It is instant; hence student can obtain a receipt as soon as they pay.

\section{Why Proposing Mobile based Payment for Nigerian Universities}

The current payment systems in Nigerian universities are human dependent with cash paid to financial institution. Most university make use of Remita where student are provided with various payment option ( Desktop App), after payment using your card details student will proceed to the bursary unit to obtain receipt to validate ones payment.

With the following steps using Remita, student still need to go to accounts dept to obtain a receipt and will be required to show a list of receipts at the end of the academic program in other to obtain a clearance certification for NYSC exercise. The draw backs experience with use of the current traditional method of payment in the university environment is pains taking and discouraging. The current system is faced with a lot of challenges including:

- The process of paying through the bank and obtaining a teller to be used for processing school payment receipt takes a lot of time that students would have used in doing some academic work.

- Since the students has to obtain their school payment receipt from the bursary department, long queue problem are experienced, having too many tellers to take record and issue receipt to student is cumbersome.

- Students complain of misplacing their teller and hence compelling the student to go through stress in other to collect another teller from bank.

- Confirmation of students payment tellers is not instant hence delay is encountered in the process.

- Error due to human deficiency can lead to issuing receipt to students with fake tellers thereby leading to loss of revenue. 


\section{Review of Previous Studies on Mobile Payment}

A clear study on the various payment platforms will converge the reality of using an ordinary mobile device whose major application is for communication between two users to making of payment. Hence mobile payment could be described as a payment method where a mobile device is needed for initiating and confirming a payment. Various terminologies have been used to define the mobile payments. The more formal formulation is stated by ${ }^{ \pm}$"A mobile payment is the transfer of an electronic means of payment from the payer to the payee through the use of an electronic payment instrument, which is a mobile device held by at least one participant, which is not bound to any place and sends and receives information over a wireless link".

In his work ${ }^{\underline{1}}$ opined that mobile payments systems (e.g. using a mobile smart phone as a digital "wallet") promise significantly increased economic productivity and personal convenience.

To establish why there will be increase usage of mobile payment among the poor populace,, in their work opined that "The technological advancement in mobile technology throughout the world is a great achievement that has affected both the rich and poor people, largely because of the prepaid model. With the advance in technology as well as the wide spread of the knowledge and the impact of mobile-money system, almost everyone within the society today are now aware and access is made available for all to have access to financial services.

According to ${ }^{2}$ they proposed the use of cryptography to secure a mobile payment system, emphasis was based on using hybrid cryptography to secure a transaction protocol. In their work, it was discovered that most protocols make use of public key cryptography for their security and also authentication is done by a single entity, and this makes the existing systems less secure since a single entity can easily be compromised leaving the whole system insecure.

$\mathrm{In}^{10}$ proposed Quick Response (QR) code android mobile system where a mobile device is used to read transaction payment details without the need of a specified piece of hardware. The proposed system constructed a mobile enabled security framework in the QR code payment system. The system proposed https based encrypted security model with QR code payment system on mobile platform.
$\mathrm{In}^{11}$ proposed also in their work proposed a modern mobile payment system based on 2-Dimentional (2D) barcodes called QR codes for mobile users to recover mobile user experience in mobile payment.

In a related work $\frac{12}{}$ proposed an NFC Payment Application capable of using NFC technology and mobile services to establish a contactless transaction system to make the payment methods easier for people. Even as NFC mobile services have great tendencies for bringing about growth, they have also caused many problems which is of a great concern to researchers which has prevented the adoption of this technology within the societies today.

\section{Proposed Mobile Payment System}

Our proposal is based on the 2D barcode technology known as Quick Response (QR) codes and Two Factor (2FA) authentication solutions. User identification will be verified at the Student Login unit, 2FA authentication is used to check the user eligibility and validates access to the App, the user will log in with e-mail address and password, using OTP (One Time Password) where as the user exceed the number of time to input correct email and password, user will have to reload the app. If the user entry was successful, an SMS token will be sent to the user phone requesting the user to enter SMS code and Student ID. If code and user ID (student) was successful, the APP will launch the user to the Dash board interface where the phone will scan the QR code and send an SMS using Unstructured Supplementary Service Data (USSD) code and payment code user will click on Generate invoice in the form QR code, the user will use a mobile generated form of the invoice. Mobile payment Gate will ensure that the USSD code tallies with the financial institution involved in the payment transaction and will ensure the transfer of money between the user financial institution and the University; mobile payment gateway debits the user and credits the University for every Payment initiated by the student. Desktop App will be installed at the bursary section to validate any payment made by the student, hence this App will be linked to the school portal and a transaction receipt will be given to the student at completion of each payment to the schools account, the user can as well check for his financial status in the Quickpay Payment System. 
Figure 1 shows the payment process of the proposed system with the overall flow of processes in the sytem. The system will be deployed with a 2FA authentication system (one time password and SMS token authentication) before the user can have access to the proposed system dashbord. On successful authentication, the user uses phone to scan the QR code and send an SMS using USSD code and payment code user will click on generate invoice in the form QR code, the user will use a mobile generated form of the invoice. Mobile payment Gate will facilitate further transaction by ensuring that the USSD code tallies with the financial institution involved in the payment transaction and will ensure the transfer of money between the user financial institution and the university is successful.

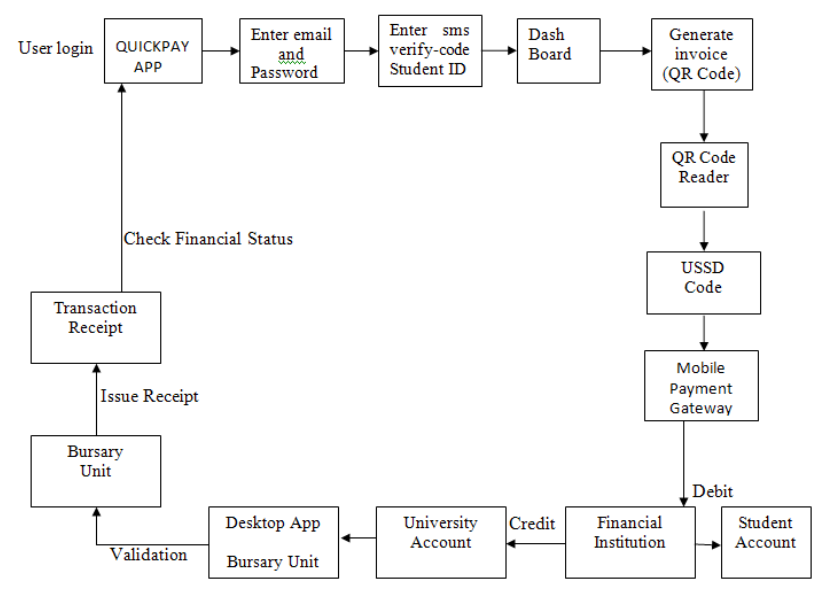

Figure 1. Payment process.

\section{Validation Process}

The validation process in Figure 2 shows that student will first log into the App using one time password and make their payment, Quickpay mobile will generate an invoice in form of code (QR code) and will scan it after which will received payment details from financial officer or institution.

Student record keeps track of every payment made by the student (database). Student can check their payment status from the Quick pay mobile using a USSD code to send instruction to the Mobile App, quick pay mobile will give a feedback as soon as transaction is completed.

There exist bursary units which will validate student payment to ensure that the transaction is successful.

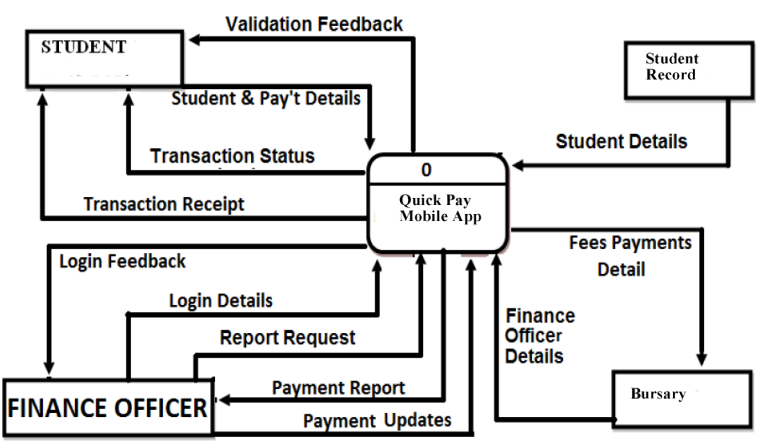

Figure 2. Validation process.

\section{Quickpay DFD}

Figure 3 shows Quickpay Data Flow Diagram (DFD) with description of all design objects that were used in developing the system. The functions of the objects are as follows:

\subsection{Process Description}

Validate Payment: This ensures that the user input matches with details of registered students as specified by the data base administrator.

Process Payment: Processes money transfer to the university.

Generate Receipt: This used as proof a payment for any transaction.

Authentication: This tern ensures that the users have access to secured system, and blocks unauthorized access.

Manage Payments Updates: Processes updates to payment records.

Generate Payment Reports: Sends feedback as requested by Finance Officers.

\subsection{Entity Description}

Student: A student for whom a payment is made. She/he also makes a payment transaction.

Finance Officer: Views reports about online payment transactions and updates payment records whenever necessary.

Bursary: Receives payment records for online transactions. Also provides information about finance officers.

Student Record: Academic Records Information System that provides information about registered students. 


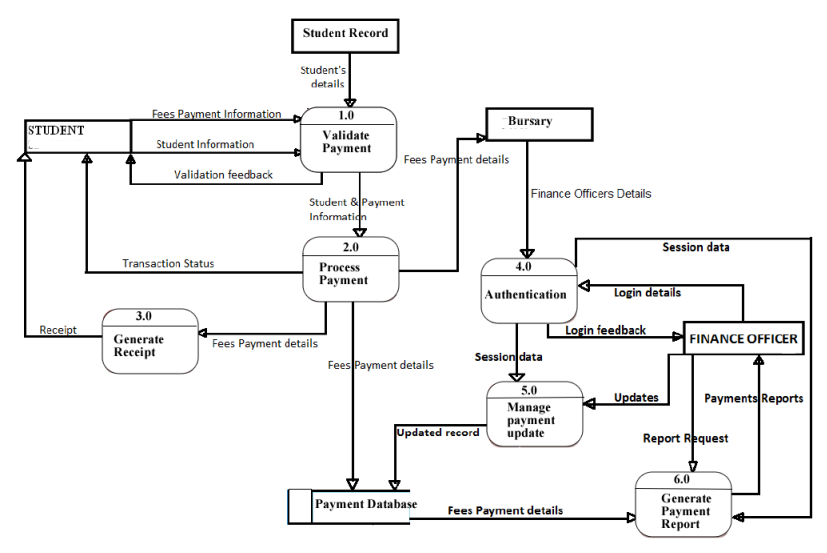

Figure 3. Quickpay DFD.

\section{User System Interaction}

The proposed system in-view is divided into several modules that needs access restrictions. Figure 4 shows user system interaction among the major actors in the proposed system and their access restrictions. The functions and roles of the major actors are represented in the diagram. It shows the basic functions carried out in the proposed system and how the interact with the entire system. The Bursary actor performs the following functions: Assign Student Reg No, Credit Account, Update Student Record and View Payment Report. The Student actor performs the following functions: Log in, Log out and Make Payment.

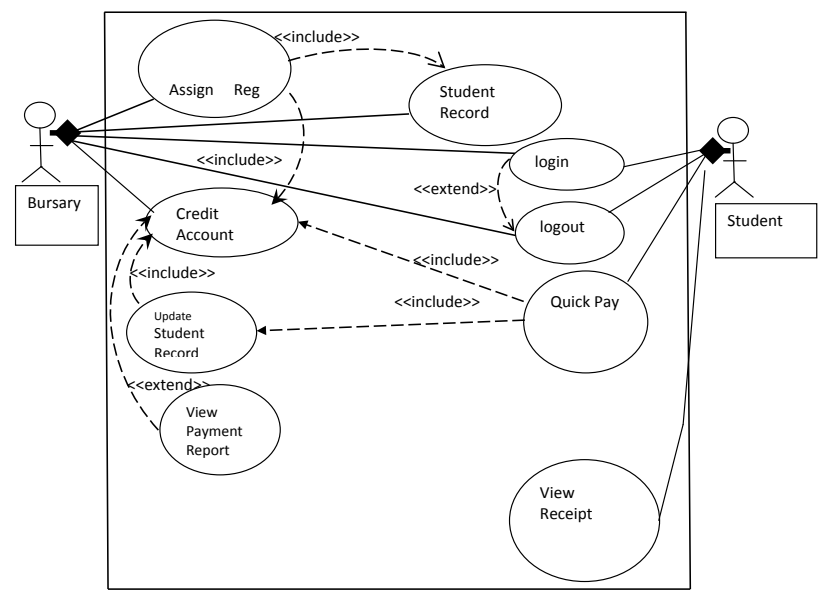

Figure 4. User system interaction.

\section{Security Model}

Security is very important when considering developing an application which will be integrated in online platform. Many security measures were analyzed but double authentication algorithm (2 factor authentication) was considered as the suitable method of security to be adopted for this application. Twice authentications could be an additional security to the systems authentication process. This ensures that the proposed system is a bit more difficult for attackers (intruders) to gain access to a person's devices or online accounts, because knowing the victim's password alone is not enough to pass authentication check. The double phase (layer) password approach is used to control access to sensitive systems as well as data. This approach is most recommendable to system that provide online service so as to effectively protect their user's credentials from being used by hackers who in one way or the other have stolen the database password or used phishing campaigns to obtain user passwords.

\section{Authentication Factors}

In terms of the application of this double layered password technique, there are several ways in which someone can be authenticated using more than one authentication method. Most of the currently used methods rely on knowledge base like the traditional password, while a two-factor authentication method uses either a possession factor or an inherent factor. Authentication factors mostly adopted are as follows:

- A knowledge factor is something the user knows, such as a password, a Pin or some other type of shared secret.

- A possession factor is something the user has, such as an ID card, a security token, a smartphone or other mobile device'.

- An inherence factor more commonly used called a biometric factor is something inherent in the user's physical self. These may be personal attributes mapped from physical characteristics, such as finger prints authenticated through a finger print reader, other commonly used inherent factors includes facial and voice recognition. It also includes behavioral biometrics, such as keystroke dynamics, gait or speech patterns.

\subsection{Workable Algorithm}

Steps:

Enter password using email address and password. Successful. 
Enter SMS token and Student ID Card No.

If Successful proceed to the Dash board else abort (exit).

Welcome to Quickpay User App interface.

Select payment option from the dashboard.

Quickpay generate an invoice in form of 2D barcode QR code.

Scan QR code using device.

Send SMS USSD Code from your Bank to initiate payment.

Account debit.

Validation processing.

Transaction successful.

Issue of receipt.

Check payment status.

End process.

A possession factor authentication method will be adopted in the design of this system whereby student will be required to use their email address and password for first time access and the second authentication will require that the student enter SMS Code and student ID number.

\section{Benefits of the Proposed System}

Quick pay will provide an additional channel for fees payment to the university online and shall integrate with the already existing systems, FINIS and ARIS. Quick pay was proposed to solve the problems associated with the current fees payment methods in the university. Quick pay is an alternative platform that enables students and their sponsors to make payment anywhere in the country. The system helps to reduce the number of students that currently miss sitting for their tests and examinations while waiting to reach bank counters to make payments. Sponsors of students, especially those abroad, will also save money and time since it will no longer necessitate them to first transfer money to students before it is paid to the university. The student will not talk about missing teller and bank delay issues.

The school will generate more revenue and have accurate financial report annually. The issue of fake receipt or teller presented by the student will be a story of the past.

\section{Conclusion}

In all, a mobile payment system (Quickpay) was proposed for Nigeria universities. The proposed system is based on QR code technology and 2FA authentication system. Quickpay mobile payment system when deployed will enhance revenue generation in Nigerian University and abort fraudster penetration into the university information database as 2 factor authentication will be deployed to secure the school database system. Interestingly, business and e-commerce platform have embraced mobile payment since it is the most convenient and reliable channel to initiate payment. Across the globe it has been widely accepted and adopted in many private and public organizations. Therefore, deploying this system will be a big relief to the universities. Quickpay mobile payment system will provide the university with a mobile version and a desktop version which will be deployed at the bursary unit for validation. Students need not to go the bank anymore as validation and bank charge will be automated. At the end of each payment the Mobile App will generate a payment receipt for the student after validation by the bursary unit.

\section{References}

1. Batchelor S. Mobile phone-enabled payment systems transformation 2008. Enterprise Development and Microfinance. 2008; 19(1):13-30. https://doi.org/10.3362/17551986.2008.003

2. Kumar B. A study on mobile payment in mobile e-commerce. International Journal of Enhanced Research in Management and Computer Application. 2013; 2(2):1-8.

3. Rouibah K. Does mobile payment technology Mnet attract potential consumers: A Kuwaiti Study. Proceedings of 18th Australasian Conference on Information System (18th ACIS); 2007. p. 199-211.

4. Mobile Payment - A comparison between the Europe and the US. 2004. https://pdfs.semanticscholar.org/ebe1/ db7281e17f6e353009efc01b00e4eacc3de3.pdf

5. Gupta S. The mobile banking and payment revolution. The European Financial Report; 2013. p. 3-6.

6. Faulds DJ, Mangold WG, Raju PS, Valsalan S. The mobile shopping revolution: Redefining the consumer decision process. Business Horizons. 2018; 61(2):232-338. https:// doi.org/10.1016/j.bushor.2017.11.012 
7. Ezell S. Explaining international IT application leaderhip: Contactless mobile payments. The Information Technology and Innovation Foundation (ITIF); 2009. p. 2-3.

8. Mobile platform for financial inclusion: The case of an unsuccessful pilot project in Brazil. 2013. https:// www.researchgate.net/publication/258820280_Mobile_ Platform_for_Financial_Inclusion_the_Case_of_an_ Unsuccessful_Pilot_Project_In_Brazil

9. Ugwu C, Mesigo T. A secured mobile payment transaction protocols for android systems. European Journal of Computer Science and Information Technology. 2015; 3(3):1-10.
10. Gresham M, Noreen S, Weston D, Dumisani S. Designing of android mobile based system using QR Code. International Journal of Innovative Research and Development. 2014; 3(11):25-33.

11. Ashvini B, Vaishali B, Dhanashri B, Patil Y. Android mobile based payment system using QR Code. International Journal of Trend in Research and Development. 2016; 3(3):231-4.

12. Pourghomi P, Muhammad QS, Gheorghita G. A proposed NFC payment application. IJACSA. 2013; 4(8):173-81. https://doi.org/10.14569/IJACSA.2013.040824 